\title{
Pelvic Organ Prolapse treatments: what would Gynaecologists opt for?
}

\section{Authors:}

Veenu Tyagi (MRCOG) ${ }^{1}$

Dr Mahesh Perera (MRCOG) ${ }^{2}$

Dr Karen Guerrero (MRCOG) ${ }^{3}$

${ }^{1}$ Subspeciality trainee urogynaecology, NHS Greater Glasgow and Clyde Southern General Hospital 1345 Govan Road

Glasgow

G51 4TF

Veenu.Tyagi@ggc.scot.nhs.uk 00441412012237

${ }^{2}$ Consultant obstetrician and Gynaecologist, NHS Greater Glasgow and Clyde Princes Royal Maternity Hospital 16 Alexandra Parade Glasgow

G16 2ER

Mahesh.Perera@ggc.scot.nhs.uk 00442115248

${ }^{3}$ Consultant subspecialist Urogynaegologist NHS Greater Glasgow and Clyde Southern General Hospital 1345 Govan Road

Glasgow

G51 4TF

Karen Guerrero@ggc.scot.nhs.uk 00441412012237

\section{Corresponding author:}

Veenu Tyagi

Veenu.Tyagi@ggc.scot.nhs.uk

01412012237

\section{Running title:}

Choice of Pelvic Organ Prolapse treatment

\begin{abstract}
A quantitative questionnaire based study was carried out to assess how acceptable the various treatments for pelvic organ prolapse are to female obstetrics and Gynaecology trainees and consultants. Uptake for vaginal pessaries was low. Majority of respondents would accept conventional surgery for primary treatment and seem to be less keen to have vaginal mesh implants for surgery. This study highlights the complexity of decision making for treatment for prolapse even for group of women who are relatively verse with the subject. It is therefore absolute essential to have insight into how women perceive the acceptability of these treatments and their associated complications.
\end{abstract}

Keywords: vaginal mesh, conventional prolapse surgery, patient choices 


\section{Introduction:}

Current treatment options for pelvic organ prolapse include pelvic floor muscle training, use of vaginal pessaries and surgery. Several surgical procedures can be used like hysterectomy, uterine or vault suspension or simple pelvic floor repairs (NICE IPG 284). There is now increasing variety of treatments available to treat Pelvic Organ Prolapse (POP) after the introduction of synthetic and biological meshes. However good studies and long term data are lacking and there is no consensus on the most effective treatment (John et al 2011).

Little is however known about women acceptability of these treatments in terms of success rates and complications. There have been surveys conducted in UK and USA looking at the current clinical practice and management of patients with vaginal pessaries (Gorti 2009). Insight into how female trainees in obstetrics and gynecology perceive the acceptability of these treatments and their associated complications would be an important addition to the existing knowledge. Do we offer all the available treatment options as gynaecologists or are we offering what we would choose for the treatment of prolapse?

\section{Aim}

We aimed to assess how acceptable the various treatments for POP are to female Obstetrics and Gynaecology trainees and consultants. We also wanted to explore perception of different treatment options and to determine what matters most when it comes to making choices about treatment.

\section{Methodology:}

We conducted a quantitative approach questionnaire based study. All the Obstetric and Gynaecology female Trainees and Consultants in West of Scotland deanery were identified via the Scottish Committee of the Royal College of Obstetrician and Gynaecologists.

Participants received a questionnaire (Appendix 1) with a stamped addressed envelope included. This questionnaire detailed nonsurgical and surgical treatments for prolapse, together with published success and complication rates. Details of age and grade were sought.

The survey was completely anonymous so that respondents could not be identified. This also meant that nonrespondents could not be approached 
again and only one mailing was carried out.

\section{Results:}

110 female gynaecologists were identified and contacted. 65 responded
(59.1\%). Majority of respondents were either consultants or senior trainees (table 1) and only 5 (7\%) had special interest in urogynaecology. Majority (26) of respondents were in the age group 30-40 (table 2).

Table 1: According to grade

\begin{tabular}{|c|c|}
\hline GRADE & NUMBER \\
\hline Consultants & 33 \\
\hline ST6-7 & 16 \\
\hline ST3-5 & 13 \\
\hline ST1-2 & 3 \\
\hline
\end{tabular}

Table 2: distribution in different range of age groups

\begin{tabular}{|c|c|}
\hline AGE GROUP & NUMBERS \\
\hline $20-30$ & 9 \\
\hline $30-40$ & 26 \\
\hline $40-50$ & 22 \\
\hline $50-60$ & 8 \\
\hline
\end{tabular}

$52(80 \%)$ respondents expected to feel better and wished symptomatic relief with better QoL with treatment. Only 13 (20\%) wished both anatomical and symptomatic improvement. None wanted only anatomical improvement.

\section{$\underline{\text { Primary treatment (Graph 1) }}$}

$39(60 \%)$ of respondents would decline vaginal pessaries. Only $12(18.4 \%)$ women accepted it as method of treatment for POP. $11(17 \%)$ were unsure about this option. 9 respondents mentioned that it will be more acceptable option if they were not sexually active at time of decision making. 
Graph 1: Showing choice of treatment for primary prolapse

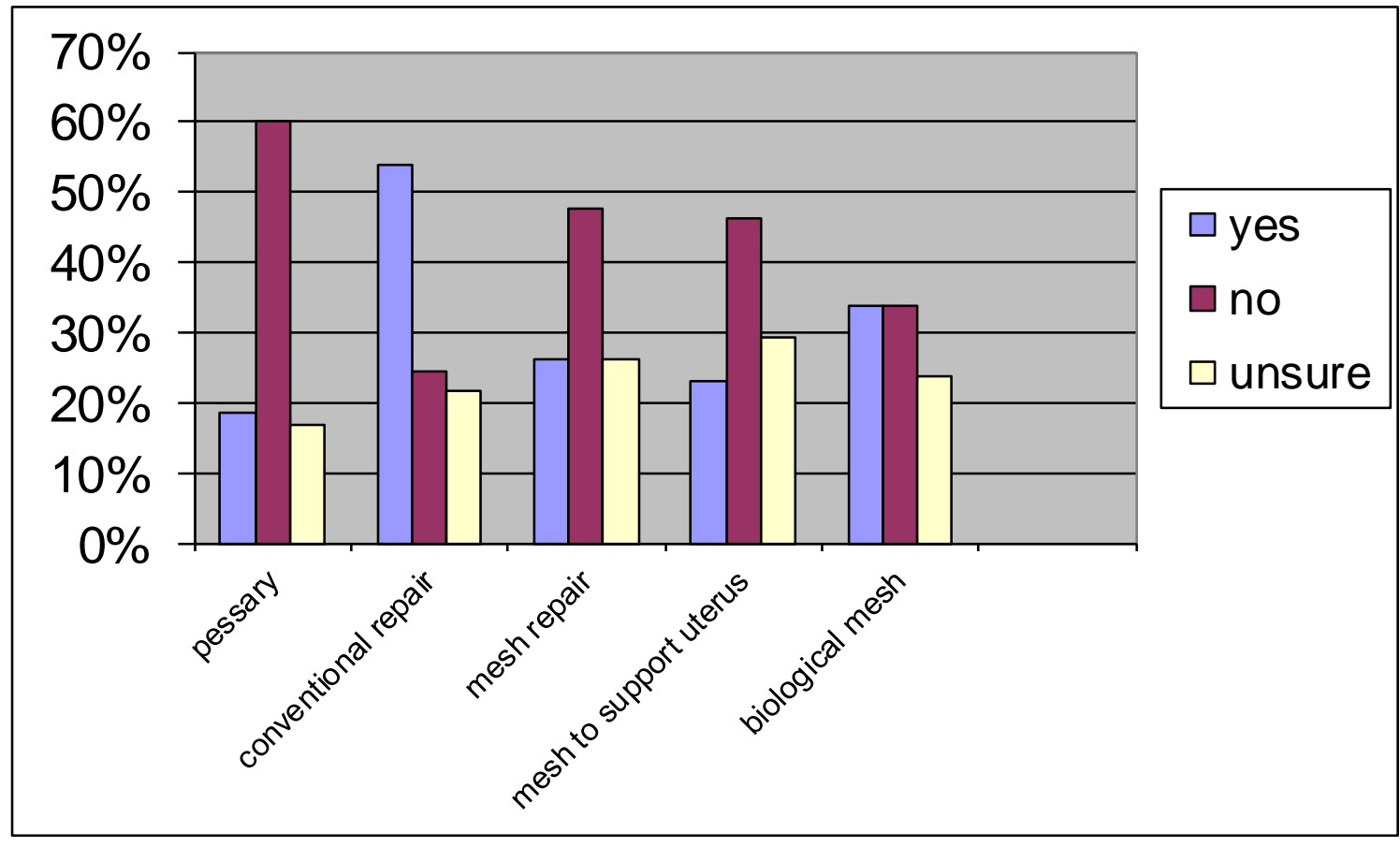

$35(54 \%)$ accepted conventional surgery i.e. repair operation +/hysterectomy as the method of operation for primary surgery. $14(21.5 \%)$ were unsure and $16(24.6 \%)$ declined the option.

Only $17(26 \%)$ accepted mesh to be used for primary surgery. $31(47.7 \%)$ declined this option for primary surgery and $17(26.1 \%)$ were unsure.

For primary surgery respondents therefore seem to be accepting of potentially higher risk of recurrence with lower incidence of complications.
$15(23 \%)$ respondents accepted surgery using mesh to support the uterus. $30(46.2 \%)$ declined and 19 (29.2\%) were unsure. Interestingly, $21(33 \%)$ accepted biological slings for repair. Fairly equal percentage declined and $22(33.8 \%)$ were unsure about this option.

\section{Surgery for recurrence of POP}

$55.4 \%$ of respondents thought that their decision would be different if it was their repeat surgery. 11 (17\%) were unsure and $12(18.4 \%)$ did not wish to change their decision from choice for primary treatment for POP. 
Mesh repair was more acceptable as a repeat procedure. For repeat surgery therefore, respondents were accepting of potentially better cure rate with lower risk of recurrence but higher risk of complications.

Respondents who said they were unsure about their choice for repeat surgery were the ones who had opted for mesh repair (synthetic or biological) for primary repair. Those who wished to not change their decision for repeat surgery had either opted for pessary in the first instance or already had opted for mesh repair for primary surgery.

In the questionnaire we did not ask whether and how much experience the doctor had with any of these treatment options.

Some of the comments by the respondents in the free text were as below.

* Complications with mesh can be severe and difficult to manage. Would like to avoid as far as possible.

* Pessary as the only choice. Will have conventional repair only if pessary fails to work.
* Definitely no mesh for primary surgery because of risks of complications.

* Seen too many mesh complications. Would like to avoid use of mesh as far as possible.

* I wouldn't have surgery for prolapse unless it is entirely necessary and somehow not very comfortable with the use of mesh of prolapse surgery.

* Would like to have an operation with maximum success and minimum complications.

\section{Discussion}

Currently available approaches to treat pelvic organ prolapse (POP) include various surgical procedures as well as conservative treatment strategies including the use of intravaginal passive devices aimed to restore the correct anatomical position of pelvic organs and therefore reduction of symptoms associated with POP (Junemann 2001, Roehl et al 2006). Vaginal pessaries have also been demonstrated to improve sexual function and therefore overall patient satisfaction (Kuhn et al 2008). 
In a UK based survey, Gorti et al 2009, looking at the current clinical practice and management of patients with vaginal pessaries, found that of all responders, $86.7 \% \quad(555 / 640$ clinicians $)$ used vaginal pessaries for conservative management of pelvic organ prolapse in their clinical practice, whereas $13.4 \%$ (85/640) opted against the use of vaginal pessaries.

Another survey of pessary use by members of the American urogynaecological society found that $77 \%$ used pessary as first line therapy for prolapse and $12 \%$ reserved it for women not surgical candidates for surgery (Cundiff 2000).

In our survey, when asked what clinician's choice as first line treatment less than 1 in 5 seems to be accepting of this option, $60 \%$ declined to use pessary. This is very different from what was found in the above 2 surveys. Some respondents mentioned that they will be more accepting to use pessary if they were not sexually active at the time of decision making.
It is quite difficult to explain why the uptake of pessary is so low in our population. Does this mean that the choice about treatment can change when it comes to making decision for clinicians themselves? Maybe because they have experienced their patient having problems with pessaries or maybe it is not treated as one off option for management of POP which clinicians would rather prefer.

When we talk about outcome for POP surgery we talk about cure. However, there is a lack of standardization of definition of disease and outcome in POP. Ideally, there are 4 main goals of pop surgery - no anatomical prolapse, no functional symptoms, patient satisfaction and avoidance of complications (Una Lee et al 2011).

In a recent article by Barber et al (2009) treating anatomy is not the most important factor for patient perception of improvement. It is the presence or absence of patient symptoms which drives patient perception of success. His data also confirmed that there is a disconnection between patient anatomy and symptoms. 
This reflects in our survey where we found none of the respondents wished only anatomical improvement. Only 1 in 5 respondents wished both symptomatic and anatomical improvement and majority wished only symptomatic improvement. This reflects a group of women who are relatively well versed with the subject. Hence, while counseling a woman prior to surgery for prolapse it is important to emphasize the fact that it is aimed to improve her symptoms rather than to an anatomical cure which would then increase patient satisfaction overall postoperatively.

In our survey, we quoted the success and complication rates of different surgeries based on data available at the time. Native tissue repair may have better success rates than previously thought. However, like repairs augmented with mesh, native tissue repairs also may be associated with complications.

A 2001 randomized trial reported success rates (based on anatomic success definitions) of only 30-46\% (Weber et al 2001). These low success rates were frequently cited as a reason why innovations such as vaginal mesh were needed to decrease failure rates. The original data from this study were recently reanalyzed using modern outcome measures and the revised success rates for the three arms of this RCT were comparable, with $89 \%$ of women having no objective prolapse beyond the hymen. Overall, only $5 \%$ of those with 1-year follow-up data were symptomatic, and there were no reoperations either for recurrence or complications at 1 year (Chmielewski et al 2011).

Since 2004, use of synthetic mesh has increased in vaginal surgery for the treatment of pelvic organ prolapse. However, concerns exist about the safety and efficacy of transvaginally placed mesh. Based on the currently available limited data, although many patients undergoing mesh-augmented vaginal repairs heal well without problems, there seems to be a small but significant group of patients who experience permanent and life-altering sequelae, including pain and dyspareunia, from the use of vaginal mesh.

Food and Drug Administration (FDA) Safety Communication released in July 2011 FDA 2011, which updates a 
2008 FDA Public Health Notification (FDA 20008), as well as published reports describing variable experience with mesh and issued the warning that transvaginal mesh products put patients at a higher risk of developing adverse effects from the procedure.

The American Urogynaecological Society in the 33rd Annual Scientific Meeting in Chicago presented the results of a November 2011 survey for surgeons (Committee Opinion 2011). This survey's purpose was to determine whether practitioners were heeding the TVM warning issued by the FDA just months before. The survey revealed that almost one-fourth of the 281 specialists surveyed were observing the FDA warning by decreasing the use of vaginal mesh to correct POP cases.

Hence, there is a significant effect of available literature and media warning on clinician's practice.

Our survey was conducted just before the FDA warning about the use of vaginal mesh came out. More and more information and data are emerging about various treatment options and the associated complications. Thus the pts as well as clinician opinion or choice or preference for treatment option may change over time.

Respondents were worried about a potentially higher complication rate with mesh and therefore were less willing to risk these for a potentially lower recurrence rate. Only 1 in 4 respondents were keen to have mesh for primary surgery. Most respondents wished a fine balance between a decreased chance of recurrence versus complications-and it was the nature of the complications and having seen and been involved in such complications which was an important factor in decision-making.

\section{$\underline{\text { Limitations }}$}

The limitations of our study need to be discussed. First, since there was no validated questionnaire available to measure treatment preference, we used self-developed questionnaires based on literature and expert opinions.

We did not actually mention what the complications could be, only gave an overall rate as quoted in the literature (Thys et al 2012). Listing the 
complications, in particular the mesh related ones, might have led our respondents to choose a different treatment options as they might have found them more or less relevant.

As our survey poses a hypothetical situation and we understand it may not truly reflect clinician's decision as various factors can influence decision making at the time. In a study (Heit et al 2003), looking at predicting treatment choice for patients with POP found that age, prior prolapse surgery, preoperative pain scores and pelvic organ prolapse severity were independently associated with treatment choices in a predictable way.

3 respondents did comment in the free text and said it would depend on how old they were at the time of decision making, whether they were fit to have surgery and if they were sexually active then.

We surveyed a rather selected group or population who are 'relatively well versed' with the subject and may have their own prefixed opinion as they deal with POP in their clinical practice. Their decision about their treatment options therefore may be skewed as they themselves were involved in complications. Also lots of respondents said they were unsure about treatment options. It may be because it was a hypothetical situation they were answering to or because it is actually quite confusing as there are variety of treatment option which are now available and they have different spectrum of advantages and disadvantages.

We did not find any similar survey in the literature where clinicians were asked about their choice of treatment if they were to have treatment for pop. Does our choice or preference affect/or can affect what we offer to our patients?? Our findings provide useful information to the clinician in managing primary and recurrent treatment of POP.

It reflects that it is very important when counseling a patient for POP surgery to give them various treatment options, advantages and disadvantages of all them and make an informed decision. In clinical practice we should incorporate patients' preferences in decision-making. This will result in improved patient satisfaction about treatment of POP. 


\section{Conclusions:}

In our study, most respondents wished more definite cure rather than conservative treatment. This may be as they were perceived as a 'one-off' treatment after which they could forget about their symptoms and carry on as before. Conservative treatment was more acceptable by some if they were not sexually active at the time of decision making.

Majority of respondents were however keen for conventional surgery without the use of mesh for primary repairs even though that meant a trade-off in terms of success rates for lower risks of complications.
This survey highlights the complexity of decision making for treatment for POP even for a group of women who are relatively versed with the subject.

\section{Acknowledgement:}

No funding was sorted for this study and there is no conflict of interest.

\section{Declaration of Interest Statement}

The manuscript has been approved by all the authors who have equal contribution and there is no conflict of interest. 


\section{Reference}

1. Barber MD, Brubaker L, Nygaard I, et al. 2009 Pelvic floor disorders network: Defining success after surgery for pelvic organ prolapse. Obstet Gynecol.;114 (3):600-9. doi: 10.1097/AOG.0b013e3181b2b1ae. [PMC free article] [PubMed] [Cross Ref]

2. Committee Opinion No. 513. 2011. Vaginal placement of synthetic mesh for pelvic organ prolapse. American College of Obstetricians and Gynecologists. Obstet Gynecol 2011; 118: 1459-64.

3. Cundiff et al 2000. A survey of use of pessary by members of the American urogynaecology society,obstet Gynaecol Jun;95:931-5

4. Chmielewski L, Walters MD, Weber AM, Barber MD. 2011. Reanalysis of a randomized trial of 3 techniques of anterior colporrhaphy using clinically relevant definitions of success. Am J Obstet Gynecol ;205:69. e1-69.e8

5. Food and Drug Administration. FDA safety communication: UPDATE on serious complications associated with transvaginal placement of surgical mesh for pelvic organ prolapse. Silver Spring (MD): FDA; 2011. Available at: http://www.fda.gov/MedicalDevices/Safet y/AlertsandNotices/ucm262435.htm.

Retrieved July 27, 2011.

6. FDA public health notification: serious complications associated with transvaginal placement of surgical mesh in repair of pelvic organ prolapse and stress urinary incontinence. Silver Spring (MD): FDA; 2008.

Available at:

http://www.fda.gov/MedicalDevices/Safet y/AlertsandNotices/PublicHealthNotificati ons/ucm061976.htm. Retrieved June 2, 2011.

7. Gorti M , Hudelist G, Simons A.2009. Evaluation of vaginal pessary managment: a UK based survey.J Obstet Gynaecol. Feb 29(2):129-31

8. Heit M, Rosenquist C, Culligan P, Graham C, Murphy M, Scott S: 2003 . Predicting treatment choice for patients with pelvic organ prolapse. Obstet Gynecol ;101:1279-1284 
9. John P.F.A Heesakkers and Mark E. Vierhout. Prolapse surgery :which technique and when? Curr Opinion Ur21:281-285

10. Junemann KP. 2001. The management of female urinary stress incontinence: The use of devices. BJU International 87:449455

11. Kuhn A, Bapst D, Stadlmayr W, Vits K, Mueller MD. 2008. Sexual and organ function in patients with symptomatic prolapse: are pessaries helpful? Fertility and Sterility

12. National Institute for Health and Clinical Excellence. Interventional Procedure Guideline 284.
13. Roehl B, Buchanan EM. 2006. Urinary incontinence evaluation and the utility of pessaries in older women. Care Management Journal 7:213-217.

14. Thys S. D, Roovers J. P., Geomini P.M., Bongers M.Y. 2012. Do Patients Prefer a Pessary or Surgery as Primary Treatment for Pelvic Organ Prolapse. Gynecol Obstet Invest ; 74:6-12

15. Una Lee and Shlomo Razশ2011. Emerging Concepts for Pelvic Organ Prolapse Surgery: What is Cure? . Curr Urol Rep. 2011 February; 12(1): 62-67.

16. Weber AM, Walters MD, Piedmonte MR, Ballard LA. 2001. Anterior colporrhaphy: a randomized trial of three surgical techniques. Am J Obstet Gynecol ;185:1299-304; discussion 1304-6. 


\section{Appendix 1}

Age $\square$ 20-30 $\square$ 30-40 $\square$ 40-50 $\square$ 50-60

\section{Grade $\square$ ST1-2 $\square$ ST3-5 $\square$ ST6-7 $\square$ Consultant $\square$ Special interest in Urogyanecology}

What do you want to achieve from your treatment?

Complete anatomical correction

Symptomatic improvement and better QoL

Both

The following statements concern a variety of treatments available for POP. Please read each one, think about whether you would accept the treatment if it were offered to you for primary surgery for prolapse and tick the appropriate box.

1. A vaginal pessary, which will support your prolapse and avoids the need for an operation, but can cause discharge and affect intercourse, and has to be changed every 4-6 months

$$
\text { Yes [ ] No [ ] Unsure [ ] }
$$

2. A repair operation +/- vaginal hysterectomy (if you have uterine prolapse) .This has an up to $30 \%$ risk of recurrent prolapse in the future.

$$
\text { Yes [ ] No [ ] Unsure [ ] }
$$

3. A repair operation using a synthetic mesh; this has a much lower risk of recurrent prolapse of $8 \%$, but can be associated with complications necessitating a second operation in $10 \%$ of women.

$$
\text { Yes [ ] No [ ] Unsure [ ] }
$$

4. A repair operation without hysterectomy, but using mesh to support the uterus. This avoids the risks of hysterectomy, but is associated with the risks of mesh; $10 \%$ of women will need a further operation due to complications of the mesh.

$$
\text { Yes [ ] No [ ] Unsure [ ] }
$$

5. A repair operation using a biological mesh. This has a risk of $18 \%$ of your prolapse recurring, but is associated with fewer complications than synthetic meshes.

$$
\text { Yes [ ] No [ ] Unsure [ ] }
$$

Reason for your choice 